Ann. Sci. For., 1987, 44 (1), 67-84

\title{
Croissance de jeunes Terminalia superba en conditions contrôlées
}

\author{
Pascale MAILlARD, Monique JACQUES, E. MIGINIAC et R. JACQUES \\ C.N.R.S., Institut de Physiologie Végétale (Phytotron), \\ F 91190 Gif-sur-Yvette
}

\begin{abstract}
Résumé
Les modalités de la croissance de jeunes Terminalia superba (arbre tropical de la famille des Combrétacées) ont été étudiées en conditions contrôlées du Phytotron à différentes températures et photopériodes. Les photopériodes les plus longues $(14$ ou $16 \mathrm{~h})$ favorisent le mieux le développement. Afin de vérifier si l'effet de la lumière sur la croissance est dû à la durée de l'éclairement ou à la quantité d'énergie lumineuse, $9 \mathrm{~h}$ d'éclairement trophique précédées par $7 \mathrm{~h}$ d'éclairement faible incandescent ont été expérimentées: l'allongement de l'axe principal est similaire à celui observé pour $16 \mathrm{~h}$ d'éclairement trophique, mais le dégagement des entrenœuds diminue et leur taille augmente. La croissance de la tige des jeunes plants est donc sensible à la durée et à l'intensité de l'éclairement. D'autre part, une différence de $5^{\circ} \mathrm{C}$ dans la température influence nettement la croissance de l'axe principal et accentue l'effet photopériodique. Les conditions les plus favorables pour le développement des plantes $T$. superba sont réunies pour une température de $27^{\circ} \mathrm{C}$ associée à une photopériode de $14 \mathrm{~h}$.

L'allongement de la tige des jeunes $T$. superba est continu à $22^{\circ} \mathrm{C}$ et périodique à $27^{\circ} \mathrm{C}$ pour des durées d'éclairement de 14 et $16 \mathrm{~h}$. Dans toutes les conditions d'environnement étudiées, l'axe principal des plantes présente une structure périodique (alternance d'entrenœuds longs et courts). Des comptages cellulaires effectués sur des entrenœuds longs et courts, ont montré que leur taille finale est fixée relativement tôt car elle est due plus à une différence de multiplication que d'allongement de leurs cellules.
\end{abstract}

Mots clés : Terminalia superba, croissance, ramification, photopériodisme, température.

\section{Introduction}

La croissance de nombreuses espèces ligneuses tempérées est sensible à l'action de facteurs du milieu comme la photopériode (NITSCH \& SomogYi, 1958) et la température (LAVARENne et al., 1975 ; MAUGET, 1983). Ces facteurs affectent de même, la croissance d'un certain nombre d'arbres tropicaux (BüNNING, 1948). En particulier, l'action de ces facteurs a été partiellement mise en évidence sur le Terminalia superba (arbre tropical

Ce travail a été réalisé dans le cadre d'une série d'études concernant le Terminalia superba engagées par le C.T.F.T. (département forestier du C.I.R.A.D.). Il a bénéficié du concours financier de l'A.T.P. C.N.R.S.I I.N.R.A./M.R.T. " Physiologie de la croissance des végétaux ligneux * (A.I.P. 51 8002) sur le thème "Déterminisme de l'induction de la floraison sur les ligneux forestiers ". 
de la famille des Combrétacées), par observation de la taille finale d'un certain nombre de ses organes aériens (Longman, 1976 ; Longman \& LeaKey, 1979).

Certaines plantes herbacées de climat tempéré, telles Vicia faba (Millet, 1970) ou Chenopodium rubrum (LeChARNy \& WAGNER, 1984), mais également des végétaux ligneux comme Quercus pedonculata (LAVAkEnNE-AllaRY, 1966) et Castanea vesca (LAVARENne et al., 1971) ou Corylus avellana (Barnola et al., 1977), peuvent présenter une croissance rythmique qui se maintient dans des conditions considérées comme favorables à la croissance, à une température constante et sous éclairement continu. Cette croissance rythmique se retrouve aussi, chez un grand nombre de végétaux ligneux tropicaux, tels Gnetum africanum (Mialoundama et al., 1984) ou Mangifera indica (PARIsor, 1985) et donne lieu à des types architecturaux particuliers. Ainsi, le Terminalia superba adulte présente un rythme morphogénétique en conditions naturelles (Fisher, 1976) et se caractérise par une architecture s'apparentant au modèle d'Aubréville (Hallé \& Oldeman, 1970).

Nous nous sommes proposés d'étudier les modalités de développement du Terminalia superba chez de jeunes plants issus de semis, en conditions expérimentales du Phytotron, afin :

- de mieux comprendre les modalités de croissance de l'axe principal sur des plantes jeunes, en conditions climatiques parfaitement maîtrisées ; plantes ;

- d'établir les conditions du milieu les plus favorables pour la croissance de ces

- de rechercher, en particulier, l'influence èventuelle de la lumière (en durée et en intensité) et de la température sur la croissance de l'axe principal et l'acquisition de l'aptitude à la ramification.

\section{Matériel et méthodes}

\subsection{Matériel végétal}

L'étude a été réalisée sur de jeunes plantes Terminalia superba obtenues par semis. Les semences, originaires de Sibiti au Congo, nous ont été fournies par le Centre Technique Forestier Tropical (C.T.F.T.) de Nogent-sur-Marne (réf. C.T.F.T. du semencier : 82/3808 N). L'étude de certaines protéines enzymatiques de jeunes plantes issues de ces semences, montre que celles-ci sont très peu polymorphes (VIGNERoN, 1984). Ce dernier caractère nous permet donc d'espérer une plus grande homogénéité des réponses face aux conditions expérimentales envisagées.

\subsection{Conditions de culture}

La germination des semences se déroule en serre, à une température de $27^{\circ} \mathrm{C}$, en éclairement naturel, augmenté par un éclairement d'appoint (mixte flurorescent et incandescent), lorsque l'énergie lumineuse descend au-dessous de $125 \mu \mathrm{E} \mathrm{m}^{-2} \mathrm{~s}^{-1}$. L'humidité relative est maintenue à 70 p. 100 . Les plantes issues du semis sont cultivées sur vermiculite et arrosées deux fois par jour : solution nutritive (mise au point par NIrSCH, décrite par Bilderling \& Lourtioux, 1976) le matin, eau déminéralisée le soir. 
Afin d'étudier l'effet de la température et de la photopériode sur la croissance du Terminalia superba, les jeunes plants âgés de 2 mois sont ensuite répartis en lots de 6 plantes et transférés en conditions contrôlées du Phytotron. Ces jeunes plants ont alors atteint une hauteur moyenne, à partir des cotylédons, de $1,2 \mathrm{~cm}$ et ils portent 4 à 6 feuilles. Deux températures $\left(22\right.$ et $\left.27^{\circ} \mathrm{C}\right)$ et quatre photopériodes à éclairement trophique constant $(9,12,14$ et $16 \mathrm{~h})$ sont utilisées. A $22^{\circ} \mathrm{C}$ une photopériode complémentaire a été expérimentée : 9 heures d'éclairement fort trophique précédées par 7 heures d'éclairement faible incandescent $(7+9 \mathrm{~h})$, de façon à vérifier si l'effet de la lumière sur la croissance est dû à la durée de l'éclairement ou à la quantité d'énergie lumineuse délivrée.

L'éclairement artificiel des salles est assuré par des tubes fluorescents (Mazda F 65, Blanc Industrie) et complété par des lampes à incandescence. La valeur de l'éclairement est de $250 \mu \mathrm{E} \mathrm{m}$ m $^{-2} \mathrm{~s}^{-1}$ au niveau des plantes pour l'éclairement fort, et de $40 \mu \mathrm{E}$ $\mathrm{m}^{-2} \mathrm{~s}^{-1}$ pour l'éclairement faible incandescent.

\subsection{Mesure de la croissance}

Chaque semaine la longueur des tiges (au-dessus de la paire de cotylédons) des plantes est mesurée au mm près, de même que celle des entrenouds constituant l'axe principal. La vitesse de dégagement des entrenœuds est définie comme étant le nombre d'entrenœuds déboités au cours du temps (ici en semaines). Les entrenœuds sont dits déboités quand ils mesurent $2 \mathrm{~mm}$.

L'apparition et le niveau d'insertion des rameaux plagiotropes sont notés.

\section{Mesure de la longueur des cellules d'entrenæuds de l'axe principal}

En fin d'expérience, à partir des plantes (âgées d'environ 6 mois) placées à $22^{\circ} \mathrm{C}$ et $14 \mathrm{~h}$ de photopériode, des répliques de surfaces épidermiques d'entrenœuds de l'axe principal ont été réalisées. La technique utilisée consiste à mouler la surface des entrenœuds à l'aide d'un matériau à base de silicone (Xantopren Plus, Bayer Dental D5090 Leverkusen). L'empreinte ainsi réalisée est dupliquée sur lame, à l'aide de vernis à ongle transparent, puis examinée au microscope optique. Le comptage des cellules s'effectue, à partir de photos prises au microscope, en trois points de l'entrenœud (haut, milieu, bas), et porte sur 15 files cellulaires chacun. Les entrenœuds pris en compte ne sont pas encore lignifiés, mais ont achevé leur croissance.

\section{Résultats}

Il apparaît en fin d'expérience que les plantes, âgées de 6 mois, présentent une répétition, le long de l'axe caulinaire, de séries d'entrenœuds longs et courts, les ramifications s'insérant généralement aux niveaux correspondant aux entrenœuds les plus courts. Toutefois, ces caractéristiques sont modulées par le comportement individuel des plantes et surtout par les conditions expérimentales. 


\subsection{Allongement de l'axe principal}

L'étude des courbes révèle l'existence à $27^{\circ} \mathrm{C}$ (fig. 1), pour toutes les photopériodes, d'une phase d'allongement faible d'environ 8 semaines, à laquelle succède une phase d'allongement plus intense. L'allongement de ces tiges est d'autant plus important que la photopériode est plus longue. Il est maximal pour une durée d'éclairement de 14 ou $16 \mathrm{~h}$ (indifféremment). De plus, pour ces photopériodes, l'allongement de l'axe principal présente une alternance de croissance lente et rapide (inflexions de la courbe aux 8 et $16^{\mathrm{e}}$ semaines de traitement). Ce phénomène est plus apparent au niveau des plantes étudiées individuellement (fig. 1a).

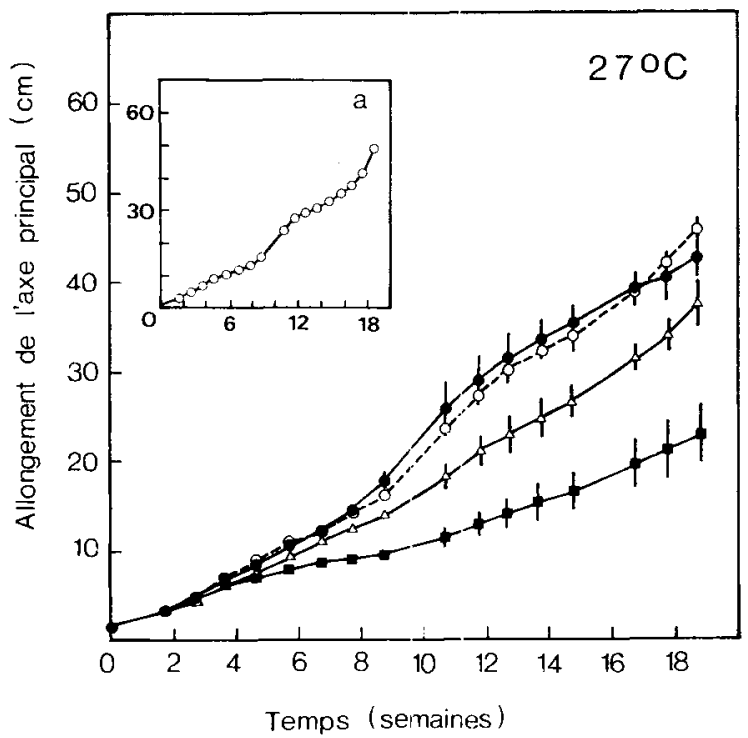

Fig. 1

Effet de la durée de l'éclairement sur l'allongement de la tige de jeunes Terminalia superba placés à $27^{\circ} \mathrm{C}: 9 h(\mathbb{0}) ; 12 h(\triangle) ; 14 h(0) ; 16 h(\bullet)$.

Effect of day-length at $27^{\circ} \mathrm{C}$ on stem elongation in young Terminalia superba plants.

(ロ) $9 h ;(\triangle) 12 h ;(0) 14 h ;(\bullet) 16 h$.

Chaque point $=$ moyenne des valeurs de 6 plantes. Les traits verticaux représentent l'écart-type Each point is the average of six measures. Vertical bars represent standard deviation.

a : Croissance d'un individu en $14 \mathrm{~h}$ ( $\mathrm{cm}$ semaine ${ }^{-1}$ ).

Elongation of the stem of a representative plant at $14 \mathrm{~h}\left(\mathrm{~cm} \mathrm{week}^{-1}\right)$.

A $22^{\circ} \mathrm{C}$ (fig. 2) comme à $27^{\circ} \mathrm{C}$, une phase de croissance lente d'environ 8 semaines précède une phase de croissance rapide pour la plupart des photopériodes étudiées. Toutefois à $22^{\circ} \mathrm{C}$ sous une photopériode de $9 \mathrm{~h}, 2$ phases successives dans l'allongement sont visibles, au cours des 9 premières semaines de croissance : la première pendant les 4 premières semaines, au cours de laquelle le taux de croissance est peu différent de celui existant au cours des sernaines 12 à 18, et la seconde pendant les 4 semaines suivantes où la croissance est sub-nulle. L'allongement des plantes augmente avec la durée de l'éclairement, néanmoins une photopériode de $14 \mathrm{~h}$ favorise 
plus l'allongement de l'axe principal qu'une photopériode de $16 \mathrm{~h}$. Il faut noter cependant que si cette durée d'éclairement de 16 heures est donnée sous forme de 7 heures d'éclairement faible suivies de 9 heures d'éclairement trophique elle a un effet similaire à une durée d'éclairement de $14 \mathrm{~h}$. L'énergie lumineuse et la durée de la photopériode ont donc une incidence sur la croissance des tiges. L'étude dés courbes individuelles des plantes pour chaque photopériode étudiée, en particulier pour $14 \mathrm{~h}$ d'éclairement (fig. 2a), ne permet pas de mettre en évidence dans la croissance des tiges des alternances de phases de croissance lente et rapide au cours du temps.

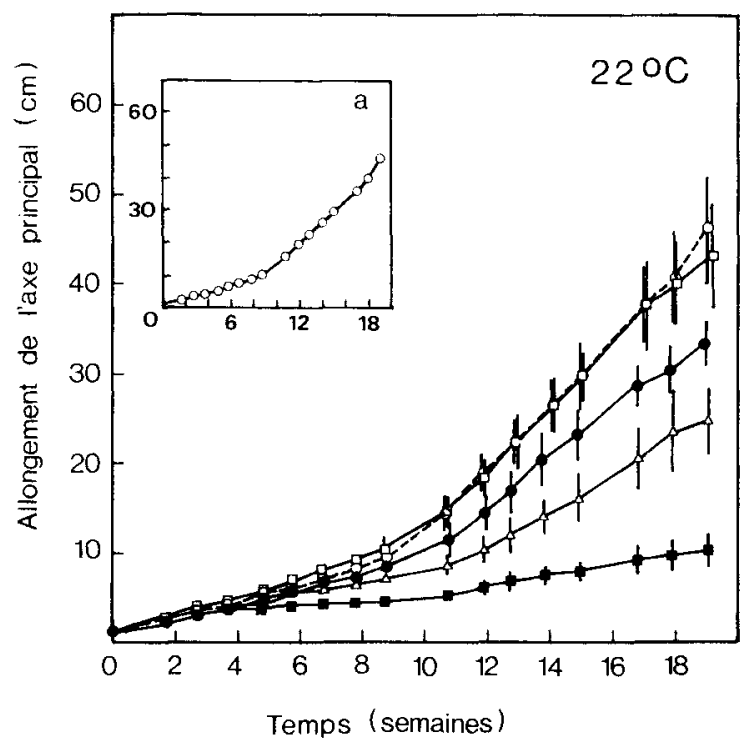

FIG. 2

Effet de la durée et de l'intensité de l'éclairement sur l'allongement de la tige

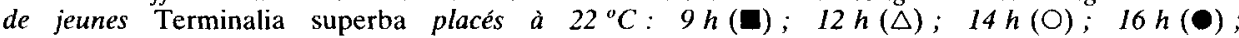
$7+9 h(\square)$.

Effect of day-length and fluence density at $22^{\circ} \mathrm{C}$ on stem elongation in young Terminalia superba plants.

(घ) $9 h ;(\Delta) 12 h ;(O) 14 h ;(\bullet) 16 h ;(\square) 7+9 h$.

Chaque point $=$ moyenne des valeurs de 6 plantes. Les traits verticaux représentent l'écart-type. Each point is the average of six measures. Vertical bars represent standard deviation.

a : Croissance d'un individu en $14 \mathrm{~h}$ ( $\mathrm{cm}$ semaine ${ }^{-1}$ ).

Elongation of the stem of a representative plant at $14 \mathrm{~h}\left(\mathrm{~cm} \mathrm{week}^{-1}\right)$.

A une température de $22^{\circ} \mathrm{C}$, la croissance de l'axe principal est plus faible que celle qui est enregistrée à $27^{\circ} \mathrm{C}$ et 9 heures d'éclairement constituent alors un facteur fortement limitant pour la croissance des tiges. Néanmoins, pour une photopériode de $14 \mathrm{~h}$ et de $7+9 \mathrm{~h}$, à $22^{\circ} \mathrm{C}$, la croissance de l'axe principal rattrape, vers la $17^{\circ}$ semaine, celle des plantes poussant à $27^{\circ} \mathrm{C}$ pour des photopériodes de durée identique. D'autre part, une température de $22^{\circ} \mathrm{C}$ met en évidence un effet favorable maximal de la photopériode $14 \mathrm{~h}$ sur l'allongement des tiges, alors qu'à $27^{\circ} \mathrm{C}$ des photopériodes de 14 ou $16 \mathrm{~h}$ ont un effet similaire. 


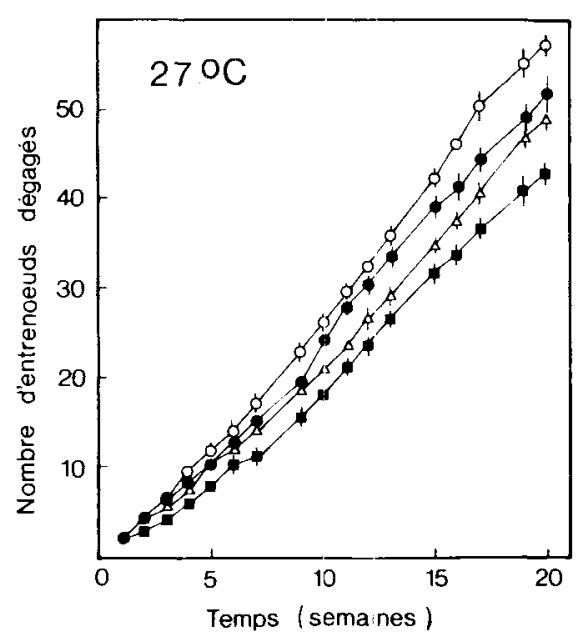

FIG. 3

Effet de la durée de l'éclairement sur le dégagement des entrenceuds de jeunes Terminalia superba placés à $27^{\circ} \mathrm{C}: 9 \mathrm{~h}(\mathbf{0}) ; 12 \mathrm{~h}(\triangle) ; 14 \mathrm{~h}(\mathrm{O}) ; 16 \mathrm{~h}(\bullet)$.

Effect of day-length at $27^{\circ} \mathrm{C}$ on internode emergence in young Terminalia superba plants.

(ロ) $9 h ;(\triangle) 12 h ;(0) 14 h ;(\bullet) 16 h$.

Chaque point $=$ moyenne des valeurs de 6 plantes. Les traits verticaux représentent l'écart-type. Each point is the average of six measures. Vertical bars represent standard deviation.

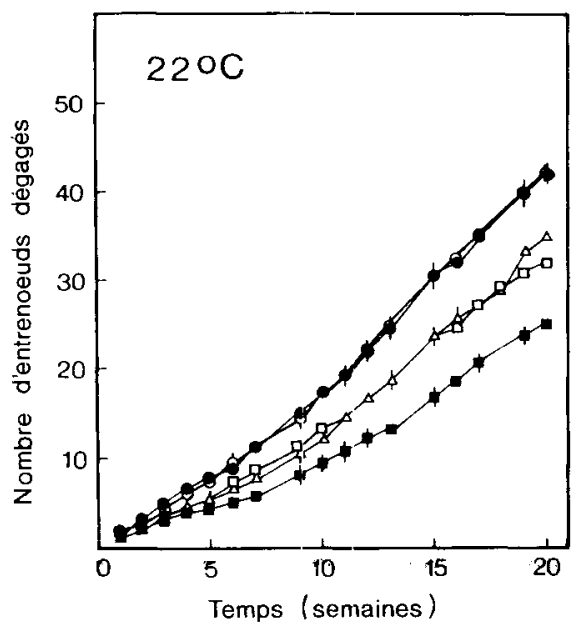

FIG. 4

Effet de la durée et de l'intensité de l'éclairement sur le dégagement des entrenceuds de jeunes

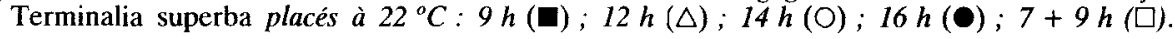

Effect of day-length and fluence density at $22^{\circ} \mathrm{C}$ on internode emergence in young Terminalia superba plants.

(Ш) $9 h ;(\triangle) 12 h ;(O) 14 h ;(\bullet) 16 h ;$ (口) $7+9 h$.

Chaque point $=$ moyenne des valeurs de 6 plantes. Les traits verticaux représentent l'écart-type. Each point is the average of six measures. Vertical bars represent standard deviation. 


\subsection{Formation des entrencuds}

A $27^{\circ} \mathrm{C}$ (fig. 3) le dégagement des entrenœuds augmente avec la photopériode, l'effet maximal étant toutefois atteint pour 14 heures d'éclairement.

Une diminution de la température de $5{ }^{\circ} \mathrm{C}$ affecte le dégagement des entrenœuds, ralenti à $22^{\circ} \mathrm{C}$ (fig. 4), et ceci d'autant plus que la photopériode est courte. Le nombre d'entrenœuds dégagés est maximal pour une photopériode de 14 ou $16 \mathrm{~h}$ indifféremment. D'autre part pour un éclairement de 16 heures $(7+9 \mathrm{~h})$, le nombre d'entrenœuds formés au cours du temps est du même ordre de grandeur que celui obtenu pour 12 heures d'éclairement et supérieur à celui atteint pour un éclairement de $9 \mathrm{~h}$. Il apparait donc que l'éclairement joue un rôle sur ce paramètre, à la fois par sa durée et par l'énergie qu'il délivre.

L'étude comparée des deux températures montre que les différences, portant sur le nombre d'entrenouds dégagés par les plantes, sont davantage marquées d'une photopériode à l'autre, à $22^{\circ} \mathrm{C}$ (fig. 4) qu'à $27^{\circ} \mathrm{C}$ (fig. 3) excepté entre 14 et $16 \mathrm{~h}$, photopériodes pour lesquelles aucune différence n'est visible.

L'étude de la longueur des entrenœuds le long de l'axe principal (profils) des plantes placées dans les différentes conditions expérimentales de température et de photopériode, révèle :

- d'une part, qu'une diminution de $5^{\circ} \mathrm{C}$ ainsi que des photopériodes courtes $[9 \mathrm{~h}$ (fig. 5) ou $12 \mathrm{~h}$ (résultat non montré)], retentissent sur la taille finale des entrencuds qui est alors plus faible. Néanmoins, pour une durée d'éclairement de $14 \mathrm{~h}$ (fig. 6) ou de $7+9 \mathrm{~h}$ (fig. 7), la taille atteinte par les entrenœuds des plantes cultivées à $22^{\circ} \mathrm{C}$ est supérieure à celle rencontrée à $27^{\circ} \mathrm{C}$ (fig. 8,9) ;

- d'autre part, l'existence de deux vagues d'entrenœuds longs très marquées aux photopériodes longues (fig. 6, 9). A $27^{\circ} \mathrm{C}$, les deux séries d'entrenœuds longs sont séparées par une plage d'entrenœuds courts plus importante qu'à $22^{\circ} \mathrm{C}$, température pour laquelle la deuxième série succède à la première presque sans transition. Cette structure périodique de l'axe principal (fig. 9), se manifeste également par un allongement de l'axe présentant deux alternances de croissance lente et rapide (fig. 1a). La structure périodique visible à $27^{\circ} \mathrm{C}$, est d'autant plus nette que les alternances de croissance sont plus marquées.

\section{Longueur moyenne des cellules épidermiques des entrenæuds longs et courts}

Les résultats indiquent que la longueur moyenne des cellules dans un entrencud est sensiblement la même que- les mesures soient faites en haut, au milieu ou en bas de l'entrenœud (tabl. 1). La variabilité constatée ne semble pas imputable à la présence d'un gradient de croissance dans l'entrenœud. Il nous paraît donc possible, à partir des données existantes, d'établir la longueur moyenne des cellules de l'entrenœud indépendamment de la zone observée. La longueur d'une cellule représentative de la longueur moyenne des cellules constitutives d'un entrenœud ayant achevé sa croissance, varie peu comparée aux différences de taille des entrenœuds. Cette longueur moyenne tend à augmenter au niveau des entrenœuds d'ordre supérieur (tabl. 2). Il semble donc que les entrenœuds les plus longs sont ceux qui présentent le plus grand nombre de cellules puisque la taille de ces dernières diffère peu que les entrenœuds soient longs ou courts, pour un même niveau de tige. 
TABLEA 1

Longueur moyenne des cellules épidermiques appartenant à 3 zones d'entrenæuds longs ou courts de l'axe principal de jeunes Terminalia superba. Les arbres sont âgés de 6 mois et placés à $22^{\circ} \mathrm{C}$ et 14 heures d'íclairement.

Epidermic cell mean length of three internode zones of long and short internodes of the main axis of Terminalia superba. The trees are 6 months old and placed at $22^{\circ} \mathrm{C}$ and $14 \mathrm{~h}$.

\begin{tabular}{|c|c|c|c|c|c|c|c|c|c|}
\hline \multirow{4}{*}{ Entrenœud court } & \multicolumn{2}{|c|}{ L } & 0,7 & 0,6 & 0,9 & 0,8 & 0,8 & 0,6 & 0,7 \\
\hline & \multirow{3}{*}{1} & h & 31 & 31 & 45 & 30 & 29 & 43 & 36 \\
\hline & & m & 29 & 29 & 45 & 30 & 29 & 36 & 31 \\
\hline & & b & 32 & 31 & 43 & 30 & 26 & 33 & 37 \\
\hline \multirow{4}{*}{ Entrencud long } & \multicolumn{2}{|c|}{$\mathrm{L}$} & 1,8 & 2,2 & 2,4 & 1,6 & 1,8 & 1,8 & 1,6 \\
\hline & \multirow{3}{*}{ I } & $\mathbf{h}$ & 32 & 36 & 45 & 43 & 40 & 32 & 33 \\
\hline & & $\mathbf{m}$ & 32 & 40 & 56 & 42 & 36 & 33 & 40 \\
\hline & & b & 36 & 40 & 56 & 36 & 43 & 36 & 34 \\
\hline
\end{tabular}

Localisation haute $(\mathrm{h})$, médiane $(\mathrm{m})$ et basale $(\mathrm{b})$ des mesures effectuées sur chaque entrencud.

$\mathrm{L}=$ longueur de l'entrencud en $\mathrm{cm}$.

$1=$ longueur d'une cellule en $\mu \mathrm{m}$.

Chaque valeur est la moyenne de la longueur des cellules de 5 rangées cellulaires.

Hight (h), medium (m) and basal (b) localization of the measurements for each internode. $\mathrm{L}=$ final length of an internode $(\mathrm{cm}) ; 1=$ mean length of an epidermic cell $(\mu \mathrm{m})$. Each value is the average of 5 cellular ranges.

\section{TABleau 2}

Variation de la longueur moyenne des cellules épidermiques des entrenauds longs et courts de l'axe principal de jeunes Terminalia superba âgés de 6 mois et placés à $22{ }^{\circ} \mathrm{C}$ et 14 heures d'éclairement.

Mean length of epidermic cells in long and short internodes of the main axis in 6 month aged Terminalia superba plants at $22{ }^{\circ} \mathrm{C}$ and $14 \mathrm{~h}$.

\begin{tabular}{l|c|c|c|c|c|c|c|c|c|c|c|c|c|c|c}
\hline \hline \\
Plante
\end{tabular}

$\mathrm{L}=$ longueur de l'entrenceud en $\mathrm{cm}$.

$1=$ longueur d'une cellule en $\mu \mathrm{m}$.

Chaque valeur est la moyenne de la longueur des cellules de 15 rangées cellulaires.

$L=$ final length of an internode $(\mathrm{cm}) ; 1=$ mean length of an epidermic cell $(\mu \mathrm{m})$. Each value is the average of 15 cellular ranges. 


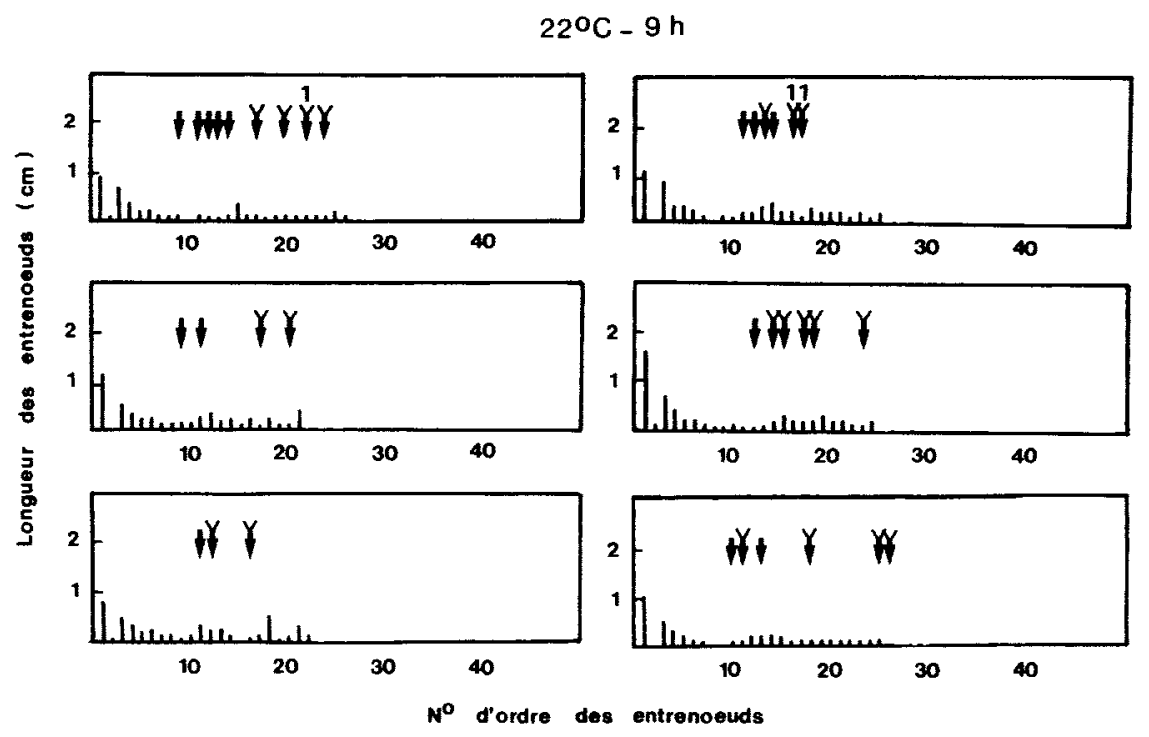

Fig. 5

Profils individuels de plantes (âgées de 6 mois) en fin d'expérience à $22^{\circ} \mathrm{C}$ et 9 heures d'éclairement, montrant les variations de la longueur finale des entrencuds de laxe principal et le niveau d'insertion des rameaux axillaires.

Individual growth patterns of 6 month aged plants at the end of the experiment at $22^{\circ} \mathrm{C}$ and $9 \mathrm{~h}$.

( 1 ) Démarrage du bourgeon axillaire sans élongation.

Development of lateral bud without subsequent elongation.

( ) Démarrage du bourgeon axillaire avec élongation.

Development of lateral bud with subsequent elongation.

$1=$ Ramifié une fois. - Axillary shoot with one ramification.

\subsection{Développement des bourgeons axillaires}

A une température de $22^{\circ} \mathrm{C}$, le ralentissement de croissance de l'axe principal s'accompagne d'une stimulation précoce de l'activité des bourgeons axillaires. Ces derniers en effet, démarrent plus tôt (un décalage d'au moins une semaine a été enregistré) qu'à $27^{\circ} \mathrm{C}$ et en plus grand nombre (fig. 5, 6), mais bien peu sont à l'origine de rameaux axillaires car ils se dessèchent et tombent.

D'autre part, nous avons pu noter à $22^{\circ} \mathrm{C}$, la présence de rameaux axillaires insérés assez bas sur l'axe principal, au niveau de la 11 ou $12^{\mathrm{e}}$ feuille (fig. 5) ; à $27^{\circ} \mathrm{C}$ les premiers rameaux apparaissent plus tard qu'à $22^{\circ} \mathrm{C}$, généralement à l'aisselle de la $15^{\mathrm{e}}$ feuille pour les photopériodes courtes (fig. 8) et de la 25 à la $35^{\mathrm{e}}$ feuille pour les photopériodes plus longues (résultats non montrés). Le niveau d'insertion des rameaux diminue également aux photopériodes courtes. Ainsi à une température de $27^{\circ} \mathrm{C}$ associée à 9 heures d'éclairement, les premiers rameaux sont présents dès la $15^{\mathrm{e}}$ feuille (fig. 8). Par contre, pour une photopériode de $14 \mathrm{~h}$ (quelle que soit la température) les premières ramifications s'insèrent à l'aisselle de feuilles de rang relativement élevé, le plus souvent vers la $30^{\mathrm{e}}$ feuille (fig. 6, 9). Pour toutes les conditions expérimentales 


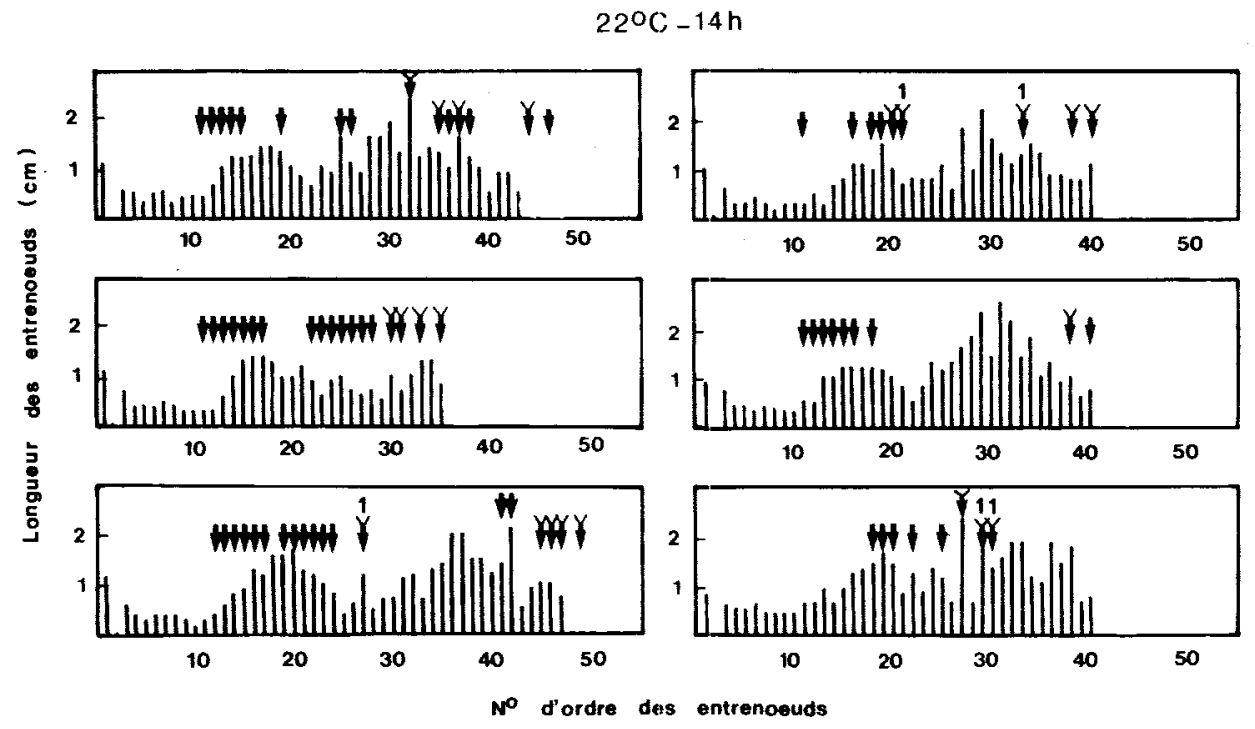

Fig. 6

Profils individuels de plantes (âgées de 5 mois) en fin d'expérience à $22{ }^{\circ} \mathrm{C}$

et 14 heures d'éclairement, montrant les variations de la longueur finale des entrenouds de l'axe principal et le niveau d'insertion des rameaux axillaires.

Individual growth patterns of 6 month aged Terminalia superba plants at the end of the experiment at $22{ }^{\circ} \mathrm{C}$ and $14 \mathrm{~h}$.

( 1) Démarrage du bourgeon axillaire sans élongation.

Development of lateral bud without subsequent elongation.

( ) Démarrage du bourgeon axillaire avec élongation.

Development of lateral bud with subsequent elongation.

1 = Ramifié une fois. - Axillary shoot with one ramification.

envisagées, les rameaux axillaires sont parfois isolés sur la tige mais le plus souvent groupés par trois ou quatre et s'insèrent généralement au niveau des plages d'entrenœuds courts.

A $22^{\circ} \mathrm{C}$, en éclairement $(7+9 \mathrm{~h})$, le démarrage des bourgeons axillaires est en règle générale toujours suivi d'élongation (fig. 6), et l'aptitude des plantes à édifier des rameaux axillaires est acquise tardivement (environ un mois après celle des plantes placées à $22{ }^{\circ} \mathrm{C}$ et à une photopériode de $16 \mathrm{~h}$ ).

\section{Discussion}

Il apparaît donc, au vu de ces résultats, que les conditions d'environnement, telles la photopériode et la température, peuvent modifier la croissance des jeunes Terminalia superba. Ainsi, une diminution de la longueur du jour ralentit fortement l'allongement 


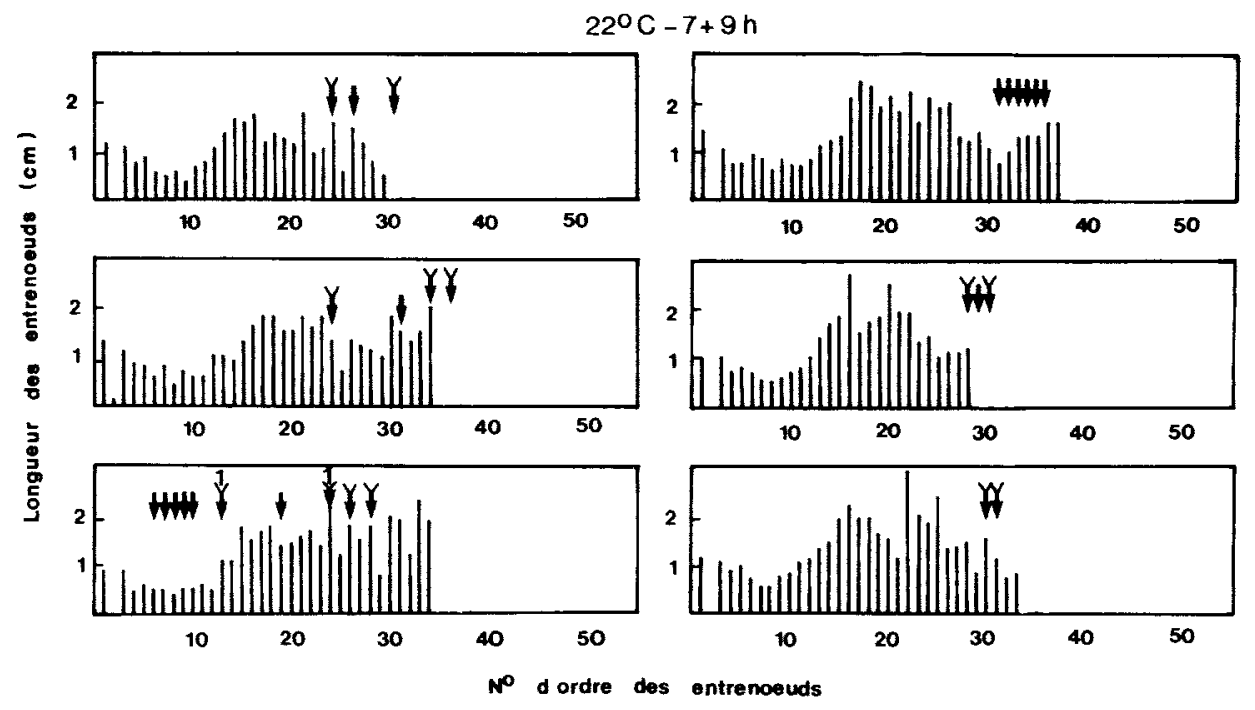

Fig. 7

Profils individuels de plantes (âgées de 6 mois) en fin d'expérience à $22^{\circ} \mathrm{C}$

et $7+9$ heures d'éclairement, montrant les variations de la longueur finale des entrenouds de l'axe principal et le niveau d'insertion des rameaux axillaires.

Individual growth patterns of 6 month aged Terminalia superba plants at the end of the experiment at $22^{\circ} \mathrm{C}$ and $7+9 \mathrm{~h}$.

( 1 ) Démarrage du bourgeon axillaire sans élongation.

Development of lateral bud without subsequent elongation.

(7) Démarrage du bourgeon axillaire avec élongation.

Development of lateral bud with subsequent elongation.

1 = Ramifié une fois. - Axillary shoot with one ramification.

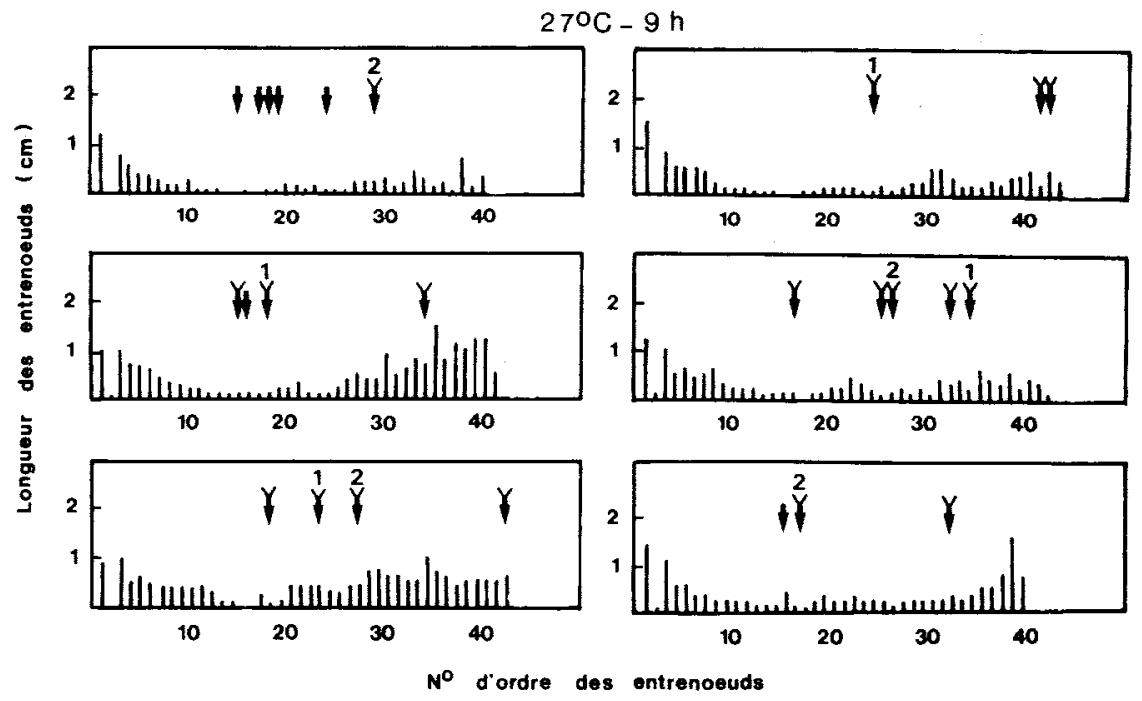

FIG. 8

Profils individuels de plantes (âgées de 6 mois) en fin d'expérience à $27^{\circ} \mathrm{C}$ et 9 heures d'éclairement, montrant les variations de la longueur finale des entrenceuds de l'axe principal et le niveau d'insertion des rameaux axillaires.

Individual growth patterns of 6 month aged Terminalia superba plants at the end of the experiment at $27^{\circ} \mathrm{C}$ and $9 \mathrm{~h}$.

( ) Démarrage du bourgeon axillaire sans élongation.

Development of lateral bud without subsequent elongation.

( $)$ Démarrage du bourgeon axillaire avec élongation.

Development of lateral bud with subsequent elongation.

1 = Ramifié une fois. - Axillary shoot with one ramification.

$2=$ Ramifié deux fois. - Axillary shoot with two ramifications. 


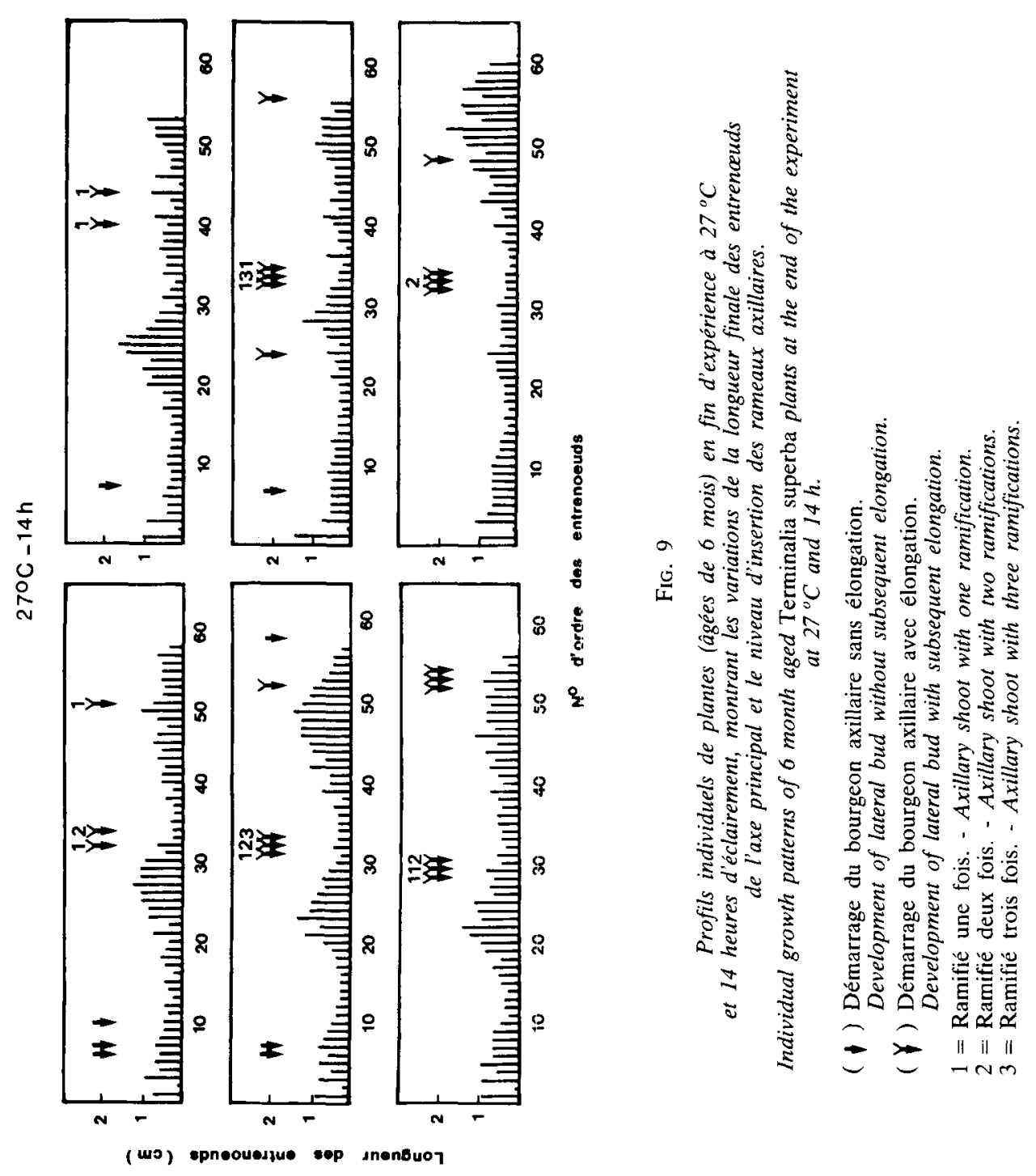


des tiges. Celui-ci est associé à un ralentissement du dégagement des entrenœuds, qui atteignent une longueur finale plus faible. Le rôle limitant des photopériodes courtes dans la croissance de cet arbre revêt un aspect intéressant pour les conditions de $22{ }^{\circ} \mathrm{C}$ et $9 \mathrm{~h}$ d'éclairement. En effet, la croissance de la tige dans les 18 premières semaines de développement de la plante, présente deux phases de croissance faible séparées par une période de croissance sub-nulle de 4 semaines (fig. 2). Celle-ci survient 4 semaines après le début de la première phase. C'est au cours de cette période que la fanaison des cotylédons survient, lorsque les plantes sont parvenues au stade de la 15 à la $17^{\mathrm{e}}$ feuille. Aussi peut-on se demander si cette période intermédiaire, qui passerait inaperçue dans les conditions de photopériode plus favorables, ne représente pas le passage de l'hétérotrophie à l'autotrophie?

Une différence de température de $5^{\circ} \mathrm{C}$ peut également affecter de façon importante la vitesse de croissance des tiges de $T$. superba et avoir une influence sur la longueur moyenne des entrenœuds. Nous retrouvons donc, en conditions contrôlées; des résultats comparables à ceux de LoNGMAN (1976). En effet, cet auteur a pu mettre en évidence l'action de facteurs du milieu sur la longueur finale moyenne des entrenœuds de $T$. superba : cette dernière augmente avec la durée du jour et sous l'effet d'une augmentation de $5{ }^{\circ} \mathrm{C}$ de la température nocturne. La croissance de l'axe principal (de même que celle de ses entrenœuds) de l'Actinidia chinensis est affectée de la même manière, par la longueur du jour et par la température, la croissance la plus faible étant enregistrée en jour court (8 heures) combiné à une température fraîche $\left(15^{\circ} \mathrm{C}\right)$ (Lonakis \& Schwabe, 1984). La croissance de l'axe principal du T. superba est d'autant meilleure que les jours sont plus longs. Cependant, à cette température de $22{ }^{\circ} \mathrm{C}$, la photopériode la plus favorable est de 14 heures et non de 16 heures, alors qu'à $27^{\circ} \mathrm{C}$ ces deux photopériodes ont un effet similaire. Ceci tend à démontrer l'existence d'une interaction entre la température et la photopériode.

La durée de l'éclairement joue un rôle non négligeable au cours de la croissance des jeunes $T$. superba; néanmoins, la valeur de l'éclairement influe aussi sur la croissance de cet arbre. En effet, l'allongement de l'axe principal peut se maintenir en jour long $(7+9 \mathrm{~h})$ même si au cours de celui-ci, seules 9 heures d'éclairement fort trophique sont assurées. Mais dans ce cas, cet allongement est dû non à une augmentation du nombre d'entrenœuds formés, mais à un allongement plus important de ceux-ci. Cet aspect photopériodique du rôle joué par la lumière sur le développement d'un certain nombre de plantes, a été démontré, en particulier sur la floraison, l'éclairement incandescent étant plus efficace s'il est donné avant qu'après l'éclairement trophique (LANE et al., 1965).

L'axe orthotrope du Terminalia adulte est un monopode à croissance rythmique ce qui confère aux branches une disposition subverticillée (Hallé \& OldEMAN, 1970). Fisher (1976) a étudié cette croissance en conditions naturelles, sur des T. superba âgés d'au moins 3 ans, en prenant comme référence la taille finale des entrenœuds et des feuilles correspondantes de l'axe principal. Il a ainsi mis en évidence un rythme morphogénétique, dont la composante est à la fois temporelle (périodes d'arrêt alternant avec des phases de croissance rapide) et spatiale. Cette dernière concerne les entrencuds et les feuilles : les feuilles dont la surface est la plus grande semblent être produites avant ou après les plus longs entrenœuds. Nous avons de même, retrouvé en conditions contrôlées du Phytotron, et sur des plantes jeunes, cette morphogénèse rythmée. Notre étude montre d'une part, que les jeunes $T$. superba édifient, au cours du temps, au niveau de la tige des structures dites périodiques (vagues d'entrenœuds longs et courts) et d'autre part que l'expression de ces structures est modifiée de façon 
notable par les conditions extérieures. Lorsque ces structures sont très marquées et que les vagues d'entrenœuds longs sont délimitées nettement par une zone d'entrenœuds courts, la croissance en longueur présente elle-même un caractère rythmique. Cette composante spatiale et temporelle se retrouve également en conditions contrôlées dans la croissance du manguier (PARISOT, 1985). La croissance rythmique du manguier présente un caractère endogène car la périodicité du rythme est de 3 à 4 semaines, différente donc de la périodicité jour-nuit du milieu qui a une influence certaine sur les plantes (Millet \& MANACHÈre, 1979).

En tout cas, il apparaît que le devenir des entrenœuds longs ou courts, dont la croissance résulte à la fois de la multiplication et de l'allongement des cellules, est fixé relativement tôt, puisque c'est la prolifération cellulaire qui est déterminante dans le processus de croissance en longueur. Ce type de croissance en longueur se rapproche donc de celui des entrenouds de tige de Vicia faba (Millet, 1970) ou de ceux du rameau annuel de Fraxinus excelsior (CotTIGNIES, 1985).

Champagnat (1983) a montré que les corrélations jouent un rôle important dans l'aptitude à faire un tronc. Celle-ci est avant tout soumise à deux processus physiologiques : la ramification acrotone de l'axe principal (l'acrotonie étant définie comme la dominance apicale du bourgeon distal capable d'inhiber la croissance des bourgeons axillaires), et le plagiotropisme des rameaux (les rameaux plagiotropes influencent la croissance des autres rameaux en auto-entretenant le «programme " plagiotrope). L'absence de démarrage d'un grand nombre de bourgeons axillaires chez le $T$. superba serait due également à un phénomène de corrélations inhibitrices : le bouturage de rejets de souches sur des arbres adultes, révèle en effet, que l'aptitude au bouturage ne diffère pas selon le niveau de prélèvement de la bouture sur le rejet (KoYo, 1985). Des facteurs extérieurs comme la lumière ou la température peuvent modifier ces phénomènes de corrélation et en particulier l'intensité de la dominance apicale (Miginiac, 1974 ; Phillips, 1975) : ces auteurs constatent d'une part, que l'inhibition corrélative des bourgeons est généralement augmentée par des éclairements de faible énergie et sous l'effet d'une augmentation de la durée de la photopériode, et d'autre part, que cette inhibition diminue aux températures fraîches. Nous observons le même type de résultats pour le $T$. superba puisqu'en éclairement de longue durée $(7+9 \mathrm{~h})$ des démarrages momentanés de bourgeons le long de l'axe principal n'apparaissent pas, et l'aptitude des plantes à édifier des rameaux axillaires est acquise tardivement. De même les rameaux axillaires apparaissent à des niveaux inférieurs sur l'axe principal lorsque la température est diminuée de $5^{\circ} \mathrm{C}$ et pour des photopériodes courtes.

Le $T$. superba présente une alternance d'entrenœuds longs et courts le long de l'axe principal, modulée par les conditions extérieures étudiées, mais toujours présente. En particulier l'influence de la lumière sur la croissance des tiges de $T$. superba, qui se manifeste selon deux modalités (durée et énergie), apparaît comme certaine. Des éclairements incandescents de faible intensité provoquent l'étiolement avec un fort allongement des entrenœuds de plantes comme le Phaseolus vulgaris (FIELD \& JACKsON, 1974) ou le Catalpa bignonioides (Downs \& Borthwick, 1956). De même, l'éclairement faible incandescent (riche en lumière infra-rouge) des conditions expérimentales $(7+9 \mathrm{~h})$ affecte nettement le nombre et l'allongement des entrenœuds formés par les jeunes $T$. superba : ceci tendrait à prouver que la qualité de la lumière joue également un rôle dans la croissance du Terminalia. Une expérimentation en cours faisant appel à des éclairements colorés tentera d'apporter des éléments de réponse à cette question.

Il est probable que la rythmicité de la croissance, qui se traduit par l'architecture très particulière du $T$. superba, ait une origine endogène. Il serait toutefois intéressant 
d'étudier la croissance de cette plante, en conditions continues, afin de vérifier cette hypothèse. D'autre part, il nous paraîtrait justifié d'aborder les phénomènes de corrélation susceptibles d'intervenir au cours de cette croissance. Nous envisageons donc notamment, d'étudier les éventuelles relations existant entre la croissance et la taille finale des entrenœuds et celles des feuilles correspondantes et de voir si les alternances de croissance de l'axe principal ont un rapport avec le développement des rameaux axillaires.

Reçu le 1 avril 1986.

Accepté le 2 juin 1986.

\section{Summary \\ Growth of young Terminalia superba in controlled environmental conditions}

In the following study, the effects of different day-lengths and temperatures on shoot growth in vegetative Terminalia superba plants (Combretaceae) have been determined.

The reaction of 2 months-old trees originating from seeds obtained at Sibiti (Congo) to various environmental conditions were studied in a controlled environment (Phytotron) at two temperatures $\left(22\right.$ or $\left.27^{\circ} \mathrm{C}\right)$ and five day-lengths $(9,12,14,16,7+9 \mathrm{~h})$.

Increasing the photoperiod resulted in an increased shoot growth (fig. 1,2). The best values of shoot extension, internode emergence rate (fig. 3,4) and final length achieved by these internodes (fig. $6,7,8,9$ ) were obtained in $14 \mathrm{~h}$ and $16 \mathrm{~h}$ day-lengths. To distinguish between true photoperiodic responses and effects of light intensity, the full intensity $9 \mathrm{~h}$ period was extended to $16 \mathrm{~h}$ by low intensity supplementary illumination $(7 \mathrm{~h}):$ the elongation of the young trunk was similar to that observed in full intensity $16 \mathrm{~h}$ photoperiod (fig. 2) but the production of internodes was reduced (fig. 4). Thus the final length of internodes was enhanced. Therefore shoot growth in Terminalia superba depends upon both day-length and light intensity.

Shoot growth was also influenced by a $5^{\circ} \mathrm{C}$ temperature difference. At $22{ }^{\circ} \mathrm{C}$ (fig. 2, 4) the growth was lower than at $27{ }^{\circ} \mathrm{C}$ (fig. 1,3) : the plants produced fewer and smaller internodes at $22^{\circ} \mathrm{C}$. However, plants growing at $22^{\circ} \mathrm{C}$ and 14 or $7+9 \mathrm{~h}$ (fig. 2) after 17 weeks reach the size of plants growing at $27^{\circ} \mathrm{C}$ and identical day-lengths. Moreover, the elongation of plants was similar at 14 and $16 \mathrm{~h}$ day-lengths at $27^{\circ} \mathrm{C}$, whereas $14 \mathrm{~h}$ photoperiod has a greater effect than $16 \mathrm{~h}$ photoperiod at $22{ }^{\circ} \mathrm{C}$ (fig. 2). It is clear that temperature and photoperiod interact, controlling the stem growth.

The development of lateral buds in young $T$. superba plants was also sensitive to day-length and temperature. Short days increased (fig. 5, 8) and long days reduced the number of growing buds (fig. $6,7,9$ ). The subsequent development of lateral buds was decreased following a temperature drop of $5^{\circ} \mathrm{C}$ (from 27 to $22^{\circ} \mathrm{C}$ ). At a given level of the stem, three or four spirally arranged lateral buds generally grow out as a closely spaced pseudo-whorl of lateral branches.

This work has shown that the trunk grew continuously at $22{ }^{\circ} \mathrm{C}$ (fig. $2,2 \mathrm{a}$ ) and periodically at $27^{\circ} \mathrm{C}$ for 14 and $16 \mathrm{~h}$ day-lengths (fig. 1, 1a). In any case, this trunk displayed alternatively long and short internodes (fig. $6,7,8,9$ ). This periodic phenomenon could not be attributed to changes in external conditions : indeed the fluctuations registered were different from periodic fluctuations of the environmental conditions. The measurement of epidermic cell mean length in long and short internodes revealed no clear difference (tabl. 1). Therefore the final length reached by the internodes is determined early and depends upon the mitotic activity inside the terminal bud.

Key words: Terminalia superba, growth, ramification, photoperiodism, temperature. 


\section{Références bibliographiques}

Barnola P., Champagnat P., Lavarenne S., 1977. « Mise en évidence d'une dormance rythmique chez le Noisetier (Corylus avellana L.) cultivé en conditions contrôlées ». C.R. Acad. Sc., Paris (série D), 284, 745-748.

BünNing E., 1948. "Studien über Photoperiodizitat in den Tropen". In : Vernalisation and Photoperiodism. Murneek A.E. et White R.O. Ed., Whaltam, Mass : Chronica Botanica, 161166.

Champagnat P., 1983. "Quelques particularités biologiques des arbres ». Bull. Soc. Bot. Fr., Actual. Bot., 130, 11-20.

CotTignies A., 1985. "Dormance et croissance active chez le Frêne (Fraxinus excelsior L.) ", Thèse Doct. Sciences, Univ. Paris, 215 p.

De Bilderling N., Lourtioux A., 1976. Quinze années de phytotronique. Dans: Etudes de Biologie Végétale. Hommage au Professeur Pierre Chouard. Jacques R. Ed., Paris, 331-341.

Downs R.J., Borthwick H.A., 1956. « Effect of photoperiod on growth of trees". Bot. Gaz., 117, 310-326.

FiEld R.J., Jackson D.I., 1974. «Light effects on apical dominance ». Ann. Bot., 39, 369-374.

Fisher J.B., 1976. "A quantitative study of Terminalia branching". In : Tropical trees as living systems. Tomlinson P.B., Zimmerman M.H. Ed., Cambridge Univ. Press, 285-312.

Hallé F., Oldeman R.A.A., 1970. Essai sur l'architecture' et la dynamique de croissance des arbres tropicaux. Monographie Bot. et Biol. Vég., Masson Ed., Paris, 178 p.

Koyo J.P., 1985. Bouturage et variabilité morphogénétique de clones de Terminalia superba Engler et Diels ou Limba du Sud-Congo, Thèse Doct. Ingénieur, Univ. Paris Sud, 146 p.

LANE H.C., Cathey H.M., Evans L.T., 1965. "The dependence of flowering in several long day plants on the spectral composition of light extending the photoperiod". Am. J. Bot., 52 , 1006-1014.

Lavarenne-Allary S., 1966. "Croissance rythmique de quelques espèces de chênes cuitivés en chambres climatisées". C.R. Acad. Sci., Paris (série D), 262, 358-361.

Lavarenne S., Champagnat P., Barnola P., 1971. "Croissance rythmique de quelques végétaux ligneux de régions tempérées cultivés en chambres climatisées à température élevée et constante et sous différentes photopériodes". Bull. Soc. Bot. Fr., 118, 131-162.

Lavarenne S., Champagnat P., Barnola P., 1975. "Influence d'une même gamme de températures sur l'entrée et la sortie de dormance de bourgeons de Frêne (Fraxinus excelsior L.) ". Physiol. Vég., 13, 215-224.

Lecharny A., Wagner E., 1984. «Stem extension rate in light grown plants. Evidence for an endogenous circadian rhythm in Chenopodium rubrum L. 》. Physiol. Plant., 60, 437-443.

Lionakis S.M., SchwabE W.W., 1984. "Some effects of day length, temperature and exogenous growth regulator application on the growth of Actinidia chinensis Planch ». Ann. Bot., 54, 485-501.

LonGMAN K.A., 1976. "Control of shoot extension and dormancy : external and internal factors ». In: Tropical trees as living systems. Tomlinson P.B. et Zimmerman M.H. Ed., Cambridge Univ. Press, 465-496.

Longman K.A., Leakey R.R.B., 1979. "Genetic and environnemental effects on shoot growth and xylem formation in a tropical tree ». Ann. Bot., 44, 377-380.

Mauget J.C., 1983. "Etude de la levée de dormance et au débourrement des bourgeons de Noyer (Juglans regia L., C. V. « Franquette") soumis à des températures supérieures à $15^{\circ} \mathrm{C}$ au cours de leur période de repos apparent ». Agronomie, 3, 745-750.

Mialoundama F., Lauzac M., Paulet P., 1984. «The periodic induction of dormancy during the rhythmic growth of Gnetum africanum Welw ». Physiol. Plant., 61, 309-313.

MiginiaC E., 1974. «Quelques aspects morphologiques, physiologiques et biochimiques de la dominance apicale". Physiol. Vég., 12, 689-720.

Millet B., 1970. Analyse des rythmes de croissance de la Fève Vicia faba $L$., Thèse Doct. Sciences, Univ. Besançon, 132 p. 
Mulet B., Manachère G., 1979. " Morphogénèse rythmée chez les vêgétaux ». Bull. Soc. Bot. Fr., 126, Actual. Bot., 51-74.

Nirsch J.P. Somogyi L., 1958. Le photopériodisme des plantes ligneuses ». Ann. Sac: Nat. Hort. Fr, 16, 466-490.

PaRisot E., 1985. Etude de la croissance rythmique chez de jeunes Manguiers Mangifera indica $L_{\text {: }}$ Thèse Doct. $3^{\text {e }}$ cycle, Univ. Clermont-Ferrand $2,156 \mathrm{p}$.

Phlulps 1.D.J., 1975. « Apical dominance », Ann. Rev. Plant. Physiol. . 26, 341-367.

VIGNERON P., 1984. Variabilité génétique des provenances ivoiriennes et congolaises de Terminalia superba Englers et Diels. Apport du polymorphisme enzymatique, Thèse Doct. $3^{\mathrm{e}}$ cycle, Univ. Paris Sud, $125 \mathrm{p}$. 